\title{
SIMULACIÓN MEDIANTE ORDENADOR DE MOVIMIENTOS BIDIMENSIONALES EN MEDIOS RESISTENTES
}

\author{
MARTÍNEZ-JIMÉNEZ, P., LEÓN ÁLVAREZ, J. y PONTES PEDRAJAS, A. \\ Colaboradores: GISBERT, M., DE LA HABA, M.A., LÓPEZ-AGUILAR, J. \\ y GARCÍA-GÁLVEZ, J.
}

\section{SUMMARY}

We have accomplished a simulation program of mechanics; it is developed in laboratory practice by students in their first years of physies studies at a University level. Its aim is a better understanding of the influence of the variable forces of friction in bidimensional movements. We have verified the effectiveness of this teaching method through the evaluation of the students' progress during the courses in which we have carried out this practice.

\section{INTRODUCCIÓN}

En la actualidad se están produciendo una serie de transformaciones en el mundo de la enseñanza y en el campo de la educación científica. Entre los numerosos factores que están influyendo en dicha transformación, cabe destacar el elevado desarrollo de las nuevas tecnologias y especialmente el uso de los ordenadores en la educación.

Los microordenadores, dentro de la enseñanza, se están utilizando con dos fines específicos:

- como sistema auxiliar del profesor en el almacenamiento y análisis de datos del proceso educativo, mejorando, por tanto, los sistemas de evaluación y recuperación (Bork 1987);

- comoinstrumento que complementa los medios didácticos tradicionales. Así, el ordenador se está usando como sistema de almacenamiento y tratamiento de datos, como controlador de los procesos experimentales, en la resolución de problemas y en la simulación de fenómenos y experiencias de laboratorio (Lowy y Rosado 1990).

El ordenador, como instrumento didáctico, se introdujo a través del paradigma conductista, que hacía especial hincapié en las posibilidades de ta enseñanza programada. Posteriormente, se han multiplicado las posibles aplicaciones del ordenador en la enseñanza, adoptando, de forma implícita, un modelo tecnológico en el que se supone que el aprendizaje puede progresar si van mejorando los medios y los procedimientos de presentar la información. Sin embargo, las numerosas investigaciones realizadas a lo largo de la última década, acerca de los llamados crrores conceptuales y concepciones alternativas (Driver et al. 1985, Hierrezuelo y Montero 1990), han puesto de manifiesto que no basta con el modelo tecnológico, sino que es necesario adoptar un enfoque constructivista acerca de los procesos de enseñanza y aprendizaje (Driver 1986, Novak 1988), propiciando la transformación de las concepciones de Ios alumnos, mediante procesos de cambio conceptual (Posner et al. 1982) y metodológico (Gil y Carrascosa 1985). En este nuevo paradigma educativo se considera que uno de los objetivos fundamentales de la enseñanza debería ser la adquisición de una noción no esterentipada de la ciencia y la familiarización de los alumnos con la metodología científica, además de mejorar la comprensión de los conceptos, la interpretación de fenómenos y la resolución de problemas (Gíl et al 1991). Conectando estas ideas con la tecnología educativa liegamos a pensar que el ordenador, adecuadamente programado, puede servir como instrumento que favorece el cambio conceptual y que ayuda al alumno a ser protagonista de su propio aprendizaje (Hewson 1990, Valente y Neto 1992).

Uno de los aspectos más interesantes del microprocesador es que permite al estudiante utilizarlo como herramienta de autoaprendizaje, mediante simulaciones que representan artificialmente situaciones correspondientes a experiencias y procesos físicos-químicos y que lo introducen en la metodología científica. 
Las principales ventajas de su utilización son (Lowy y Rosado 1990): a) representación de situaciones, de difícil implementación práctica, que requieren equipos costosos y complejos o de manipulación peligrosa; $b$ ) utilización de modelos parciales del mundo real o idealización de las condiciones de un experimento; $c$ ) manipulación y control exacto de variables.

La simulación de fenómenos tiene unas aplicaciones muy importantes en la enseñanza de la física, tales como el análisis de movimientos, dibujo de trayectorias, descripción vectorial de los fenómenos fúsicos, formación de imágenes en óptica geométrica, fenómenos ondulatorios, sistemas eléctricos y electrónicos, procesos atómicos y nucleares, etc. (Hurley 1986, Franco 1991, Martínez et al. 1992, Miro et al. 1990, 1991).

En el campo específico de la mecánica, existen variedad de programas relacionados con el estudio cinemático y dinámico del movimiento (Borghi et a1. 1987, Martín et al. 1988, Hewson 1990, Valente y Neto 1992). Por otra parte, también se han desarrollado diversos trabajos en los que se destaca la utilización práctica del ordenador como herramienta para la enseñanza experimental (Gorga et al. 1989).

Nuestra línea de trabajo trata de proyectarse en este marco educativo, donde se presta atención al desarrollo del material didáctico, utilizando como herramienta el ordenador para la enseñanza de la física y, en segundo Iugar, se empieza a hacer un esfuerzo por conectar este proceso de elaboración de software educativo con las nuevas ideas que han surgido en la didáctica de la ciencias. La mayoría de los programas de simulación o de tipo tutorial que se emplean en la enseñanza asistida por ordenador vienen a ser un vehículo para presentar información al alumno a través de la pantalla, pero un enfoque más innovador debería tener en cuenta otros aspectos tales como las preconcepciones erróneas de los atumnos, las destrezas intelectuales requeridas para procesar la información que recibe, el análisis de los variables que influyen en una situación física, etc.

En relación con las ideas apuntadas anteriormente, el trabajo que aquí se presenta aborda la simulación del movimiento de proyectiles, que es un problema de interés para clarificar los principales conceptos de cinemática y dinámica (trayectoria, vector de posición, velocidad instantánea, aceleración, fuerza), donde existen numerosas confusiones y crrores conceptuales por parte de los alumnos de nivel secundario y universitario (Gil y $\mathrm{Ca}$ rrascosa 1985, Hierrezuelo y Montero 1990), pero además se relaciona con la problemática del control de variables, al analizar la influencia del medio material resistente donde se realiza el movimiento. Éste es un aspecto que no ha sido tratado en otros programas sobre simulación de movimientos y que tiene cierto interés en la física de nivel universitario.

Desde el punto de vista didáctico, el material informático se complementa con un programa-guía de actividades que orienta la acción del alumno durante el desarrollo del trabajo.

\section{DESCRIPCIÓN DEL INSTRUMENTO DIDACTICO}

\section{Aspectos generales}

El estudio experimental del movimiento bidimensional de cuerpos sometidos a fuerzas de rozamiento no constantes (medios resistentes) presenta ciertas dificultades de realización práctica, ya que requiere el uso de equipos didácticos experimentales no disponibles en muchos laboratorios de física. Sin embargo, el análisis de las características de estos movimientos es una actividad de gran valor educativo, pues obliga a tratar con fuerzas que son función de la velocidad del propio movimiento y a estudiar la influencia de las distintas variables que lo afectan.

Por tales motivos, se ha elaborado un programa interactivo que simula el comportamiento de un sistema experimental, en el que se lleva a cabo el estudio de la inffuencia de fuerzas de rozamiento variables en el movimiento bidimensional de diferentes cuerpos (Marion 1975). El programa se ha diseñado en lenguaje C (Hancock y Krieger 1987), para un ordenador PC compatible con pantalla hércules o emulador de ésta.

Dicho programa se ha utilizado, como un ejemplo de experiencia de laboratorio simulada por ordenador, con alumnos de primer curso de Ingeniería Técnica en dos años consecutivos. En el proceso de experimentación educativa, se ha estudiado la influencia que la realización de este tipo de prácticas tiene en el aprendizaje de la física. Para ello, se han comparado los resultados de las calificaciones que los alumnos de estos dos últimos cursos han obtenido, en una prueba sobre los contenidos de cinemática tratados en la experiencia, con las de los alumnos de los tres cursos anteriores (1988-1990), observándose una mayor comprensión de los conceptos físicos y un manejo más correcto de los algoritmos matemáticos.

\section{Fundamento físico del problema: análisis del movimiento}

La importancia que la resistencia del aire tiene en el movimiento de los cuerpos es evidente, en especial para el cálculo de las trayectorias de los proyectiles. Con frecuencia en la descripción del movimiento, basta suponer que la fuerza de rozamiento es proporcional a alguna potencia de la velocidad, y en particular esta potencia vale i para velocidades inferiores a $24 \mathrm{~m} / \mathrm{s}$ y superiores a $660 \mathrm{~m} / \mathrm{s}$ (Marion 1975).

Se examina a continuación el movimiento de un proyectil en la atmósfera, donde el aire origina una fuerza de resistencia que se supone proporcional a la velocidad instantánea. El propósito de este desarrollo es determinar la disminución del alcance por efecto de la resistencia del aire, así como la influencia que sobre esta disminución tiene la velocidad inicial, tanto en módulo como en dirección.

Si se consideran los parámetros iniciales $V_{0}, \alpha, k$, donde $\mathrm{V}_{0}$ es el módulo de la velocidad inicial, $\alpha$ es el ángulo del 
vector velocidad con respecto a la horizontal, y $\mathrm{x}$ es la constante de resistencia del aire, la descripción del movimiento puede hacerse a partir de las siguientes ecuaciones diferenciales:

$$
m \ddot{x}=-k m \dot{x} ; \quad m \ddot{y}=-k m \dot{y}-m g
$$

cuyas soluciones son:

$$
\begin{gathered}
\mathrm{x}=-\frac{v_{o x}}{k}\left(1-e^{-k t}\right) \\
\mathrm{y}=-\frac{g t}{k}+\frac{k v_{o y}+g}{k^{2}}\left(J-e^{-k t}\right)
\end{gathered}
$$

El alcance del proyectil ( $R$ ) se determina calculando, en primer lugar, el tiempo $T$ (tiempo de vuelo) necesario para que la partícula recorra toda la trayectoria y sustituyendo éste en la ecuación 1 correspondiente al desplazamiento.

$$
T=\frac{k v_{o y}+g}{g k}\left(1-e^{-k t}\right)
$$

La complejidad del procedimiento se presenta en la resolución de la ecuación 3 , que es trascendente.

Los métodos matemáticos utilizađos tradicionalmente para la obtención de la expresión analítica del tiempo de vuelo del proyectil son dos:

a) Gráfico: en el cual el tiempo de vuelo se obtiene como solución gráfica del sistema de ecuaciones:

$$
y_{1}=T \quad y_{2}=\frac{k v_{a y}+g}{g k}\left(1-e^{-k t}\right)
$$

como se observa en la figura 1.

Figura I

Método grâficico de resoluciớn dej tiempo de vueto. $\mathrm{T}=\left\{\left(\mathrm{kv}_{\mathrm{oy}}+\mathrm{g}\right) / \mathrm{gk}\right\}(1-\exp (\mathrm{kt})\}$

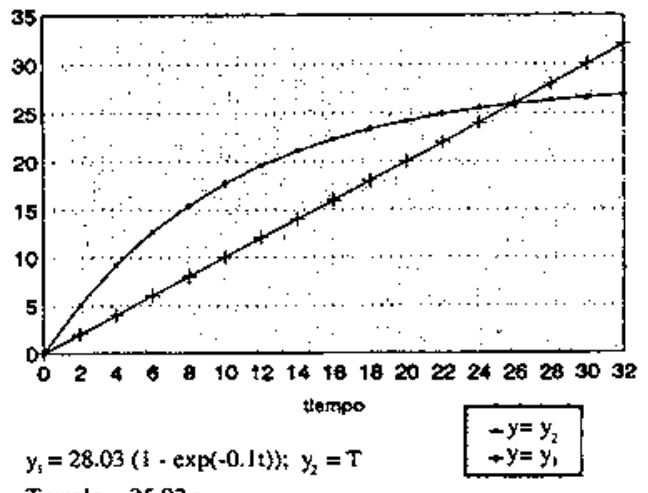

$T$ vaelo $=25.92 \mathrm{~s}$ b) Aproximado: en el cual, mediante un desarrollo en serie, y tomando límites cuando $\mathrm{k}$ tiende a cero, se obtienen las aproximaciones lineales siguientes (4 y 5).

$$
T \cong 2-\frac{v_{o v}}{g}-\left(1-\frac{k v_{u y}}{3 g}\right)
$$

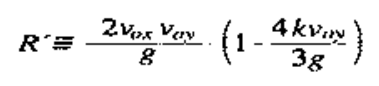

Como se puede observar en la figura 2, en la que se ha representado $\Delta R / R$ frente a la constante de rozamiento para Ia aproximación lineal y para los valores reales, đicha aproximación sólo es válida para valores de constantes iguales o inferiores a 0,01; por encima de éste la desviación del alcance real respecto al calculado se incrementa rápidamente $\mathrm{y}$, por tanto, la aproximación lineal deja de ser factible.

\section{Descripción del simulador}

\section{Estructura interna}

El objetivo del programa desarrollado es implementar mediante el ordenador la variación que sobre la trayectoria del movimiento parabólico ideal se produce cuando

\section{Figura 2}

Desviacín del incremento del alcance aproximado respecto del real

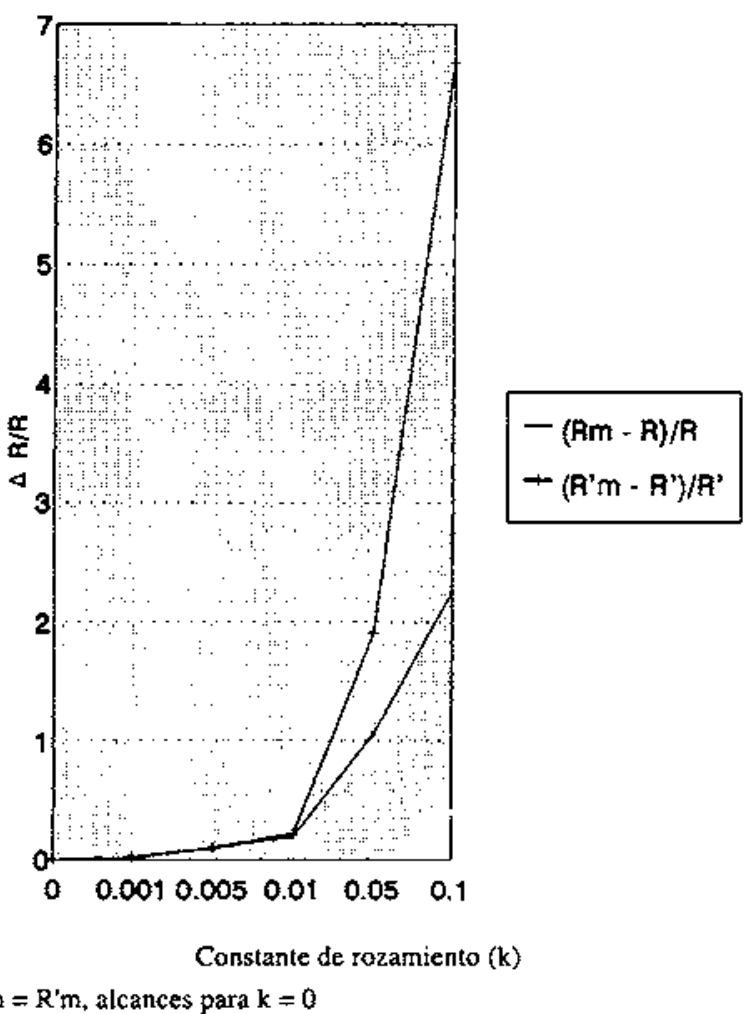


Figura 3

Diagramas de flujo: a) General, b) Cálculos generales, c) Cálculos de tiempo.

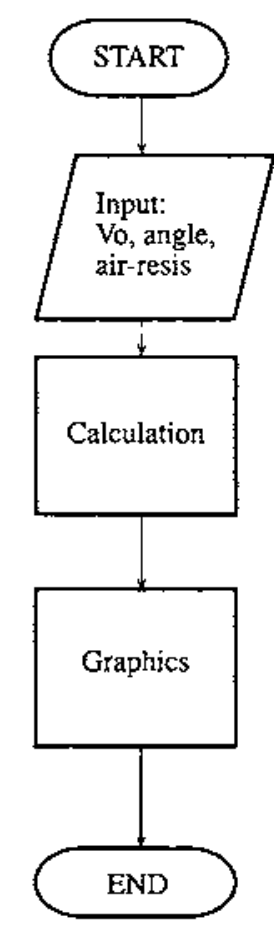

General Flow Chart $3 a$

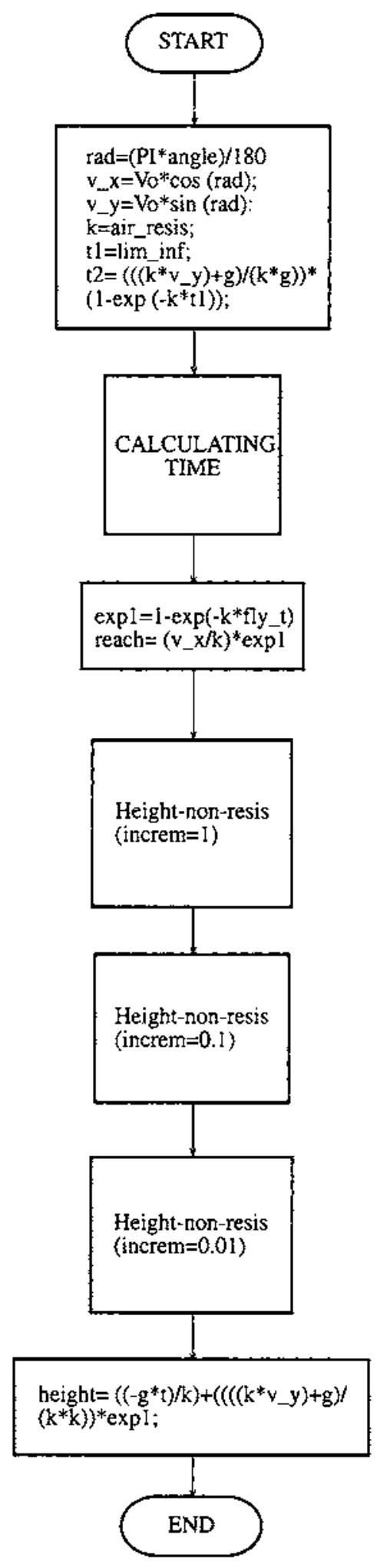

Calculation Flow Chart

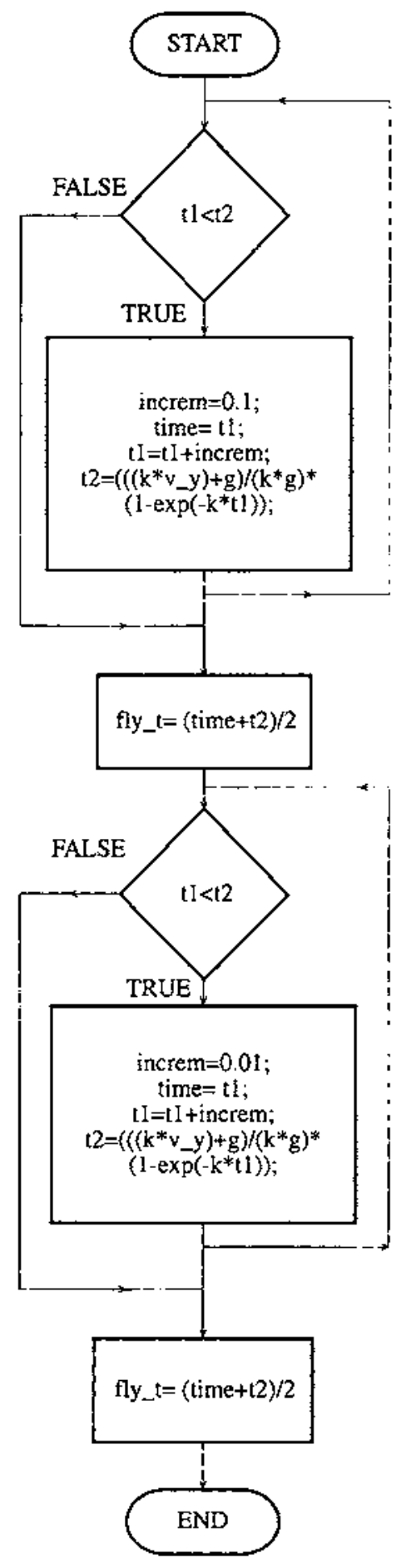

Calculating Time Flow Chart

$3 c$ 
sobre el proyectil en movimiento actúan fuerzas resistentes al mismo; para ello, es preciso determinar los resultados analíticos del alcance y de la altura para diferentes valores iniciales de velocidad, ángulo y constantes de resistencias del medio, así como representar gráficamente el movimiento considerado. El estudio se realiza para cinco proyectiles diferentes, comparándose los resultados obtenidos en cada uno de ellos.

Con este fin, el programa principal, llamado PR, consta de dos etapas fundamentales, tal como se puede observar en la figura 3a donde se muestra el diagrama de flujo general, correspondiendo la primera al cálculo de los resuftados numéricos y la segundia a la representación gráfica. En el apartado 36 de dicha figura se ha desarrollado el diagrama de flujos correspondientes a la realización de los cálculos generales; y en la figura $3 c$ se refleja el proceso de determinación del tiempo de vuelo del proyectil, que corresponde a la tercera etapa de los cálculos generales.

Los datos necesarios para el trazado de la trayectoria, cuando el proyectil se mueve en un medio no resistente, se obtienen a través de las ecuaciones correspondientes al movimiento parabólico. Por el contrario, los puntos que configuran la trayectoria del movimiento en un medio resistente se determinan mediante las ecuaciones
1, 2 y 3. Tal como se observa en el diagrama de flujo correspondiente al cálculo del tiempo de vuelo (fig. $3 \mathrm{c}$ ), el método utilizado para la resolución de la ecuación trascendente 3 es iterativo y se basa en el método gráfico de cálculo comentado en el apartado 2.2. En él, el programa toma pequeños incrementos en la variablc independiente «t» de la ecuación $y_{2}$, hasta que el resultado de ésta coincide con el correspondiente a $y_{1}$. Dichos incrementos son ajustados por el programa, de manera que el error en la determinación de $t$ sea mínimo.

\section{Visualización y representación}

El programa interactivo desarrollaco tiene la característica fundamental de su fácil manejo, no requiriéndose tener conocimientos informáticos para poder visualizar la simulación.

Mediante un sistema de ventanas (pull-down) el programa permite el estudio simultáneo de cinco cuerpos con diferentes parámetros de entrada. La introducción de los datos se realiza eligiendo uno de los cinco proyectiles, lo cual queda especificado en una barra que aparece en la parte superior de la pantalla por la que nos podemos mover libremente utilizando para ello los cursores correspondientes a las flechas.

Resultados del estudio comparativo del movimiento bidimensional de cinco proyectiles en diferentes medios resistentes.

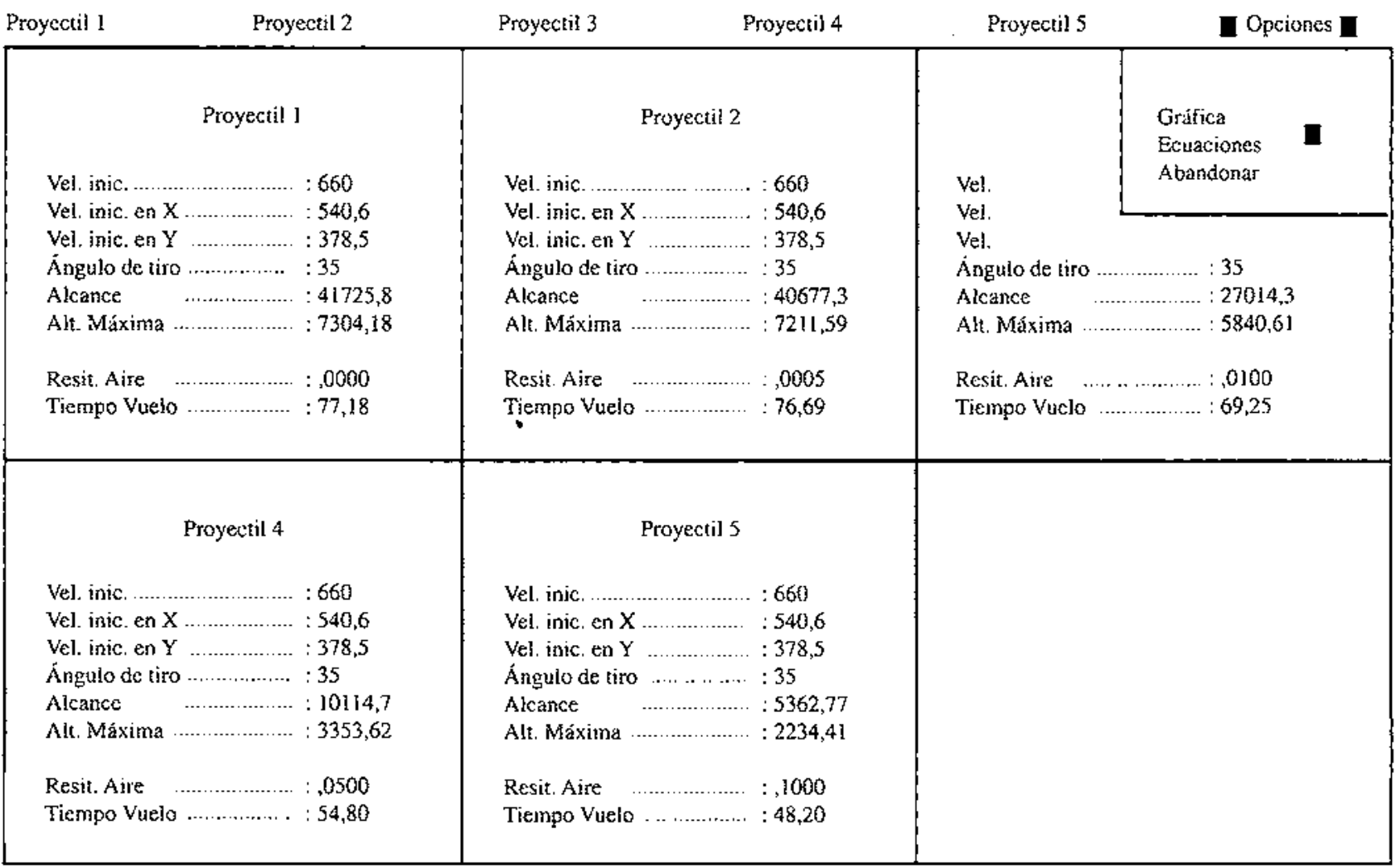


El estudio del movimiento bidimensional de cada proyectil se realiza independientemente dentro de su ventana correspondiente. Una vez introducidos los datos iniciales, los resultados correspondientes a la determinación del alcance, altura y tiempo de vuelo de cada uno de ellos se muestran, así mismo, dentro de dicha ventana. Cuando se completa todo el proceso, y tal como se observa en la figura 4 , en una misma pantalla aparecen los resultados correspondientes a cada estudio, pudiéndose determinar la influencia de las distintas variables en el movimiento. Posteriormente, las trayectorias se observan, simultáneamente, dentro del apartado «opciones».

\section{IMPLEMENTACIÓN DIDÁCTICA}

\section{Objetivos programados}

Siguiendo el modelo de las prácticas experimentales desarrolladas y realizadas en el laboratorio, seis son los objetivos programados con la implementación práctica de la simulación propuesta, agrupados, a su vez, en tres apartados diferentes:

a) Comprender la influencia que ejercen las diferentes variables físicas sobre la trayectoria del movimiento bidimensional.

1. Variación de la trayectoria en función de la resistencia del medio, $\kappa$.

2. Variación de la trayectoria en función del módulo de la velocidad inicial.

3. Variación de la trayectoria en función del ángulo inicial que forma el vector velocidad con la horizontal.

b) Estudiar diferentes métodos de resolución de cálculo númerico en la determinación del tiempo de vuelo, y como consecuencia del alcance y altura del movimiento:

\section{Método aproximado.}

5. Método gráfico.

c) Evaluar y comparar los resuitados obtenidos mediante los métodos matemáticos tradicionales con los correspondientes de la simulación (objetivo 6).

\section{Metodología de trabajo}

Con el fin de alcanzar los objetivos propuestos inicialmente, se entrega a cada alumno un programa guía, en el que, después de hacer una introducción teórica del fundamento físico de la simulación y del mođo de operación del programa, se le guía en el proceso de realización de dicha simulación. Este último se estructura en tres apartados fundamentales:

a) influencia de la resistencia del medio (fig. 5a). b) influencia de la variación de la dirección de la velocidad inicial (fig. 5b).

c) influencia del módulo de la velocidad inicial.

En cada uno de ellos, el alumno ha de explicar el fundamento fúsico de dicha variación y, a la vez, ha de comparar los resultados obtenidos mediante la simulación con los calculados a partir de los métodos gráficos y de aproximación tineal.

\section{Figura 5}

Trayectorias de los proyectiles:

a) Influencia de la constante de rozamiento.

b) Influencia del ângulo inicial.
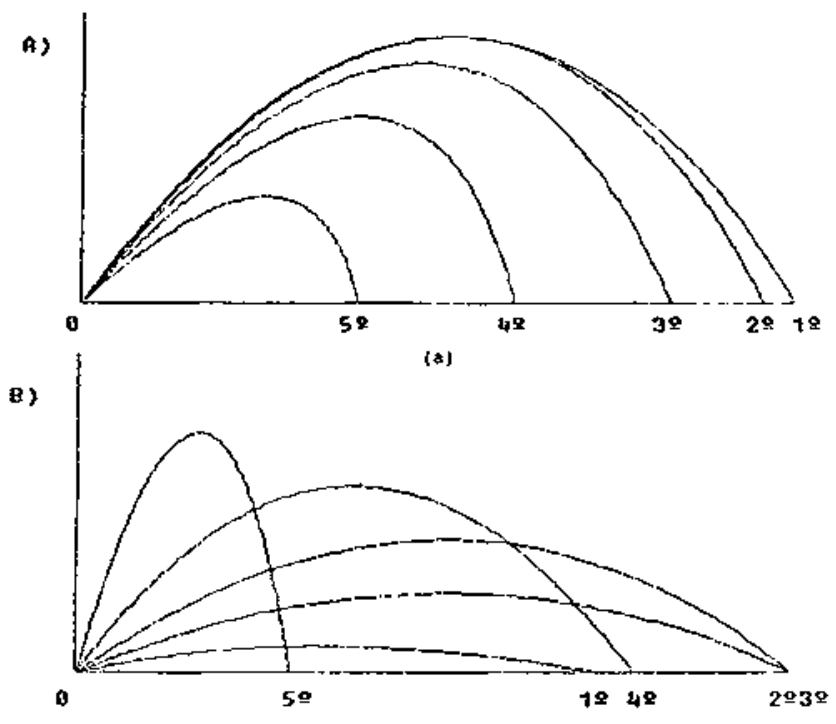

(b)

\section{Método y criterios de evaluación}

A fin de evaluar el grado de satisfación en los logros de los objetivos propuestos, se invita al alumno a entregar una memoria de prácticas en la cual han de mostrar no sólo los resultados obtenidos sino también las técnicas de cálculos utilizadas y la determinación de la exactitud y precisión de los métodos por comparación con los resultados exactos procedentes de la simulación. Así mismo, se le piden las respuestas a una serie de cuestiones conceptuales referentes al fundamento físico e influencia de las diversas variables que afectan a los movimientos estudiados.

Los resultados obtenidos se muestran en la figura 6 (año 1991) y en el diagrama de barras correspondiente a la figura 7 (año 1992), en el que se han representado las frecuencias de cada una de las calificaciones y para cada objetivo.

Se observa que todos los objetivos propuestos inicialmente se aicanzaron plenamente, siendo muy bajo el 
Figura 6

Valoración de los objetivos docentes propuestos en la simulacion

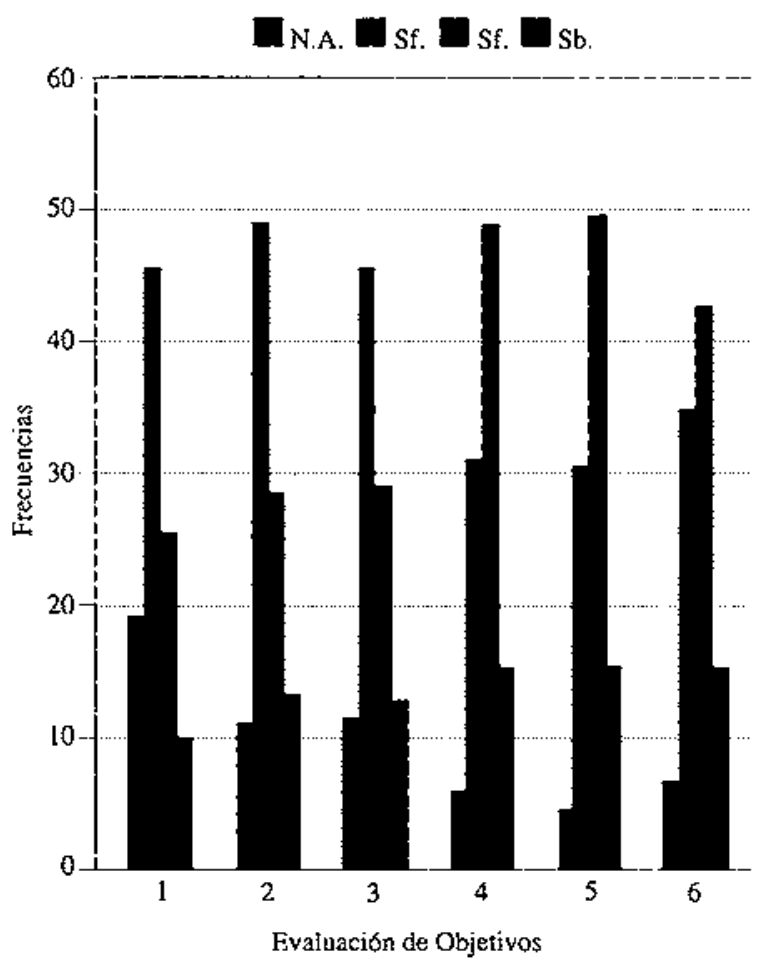

Octubre de 1991

Mucstra de 65 alumnos

tanto por ciento de alumnos que no han logrado el mínimo exigido. Por otra parte, se pone de manifiesto que, mientras los objetivos conceptuales tienen su máximo de frecuencias en la calificación de suficiente, los correspondientes al método operacional lo tienen en la de notable.

De todo lo expuesto, se puede concluir que los alumnos aprenden con más facilidad a operar matemáticamente que a desarrollar su capacidad de deducción.

\section{INFLUENCIA DEL INSTRUMENTO DI- DACTICO UTILIZADO: ANALISIS DE RESULTADOS}

Con el fin de determinar la influencia que este instrumento didáctico ejerce en la calidad de la enseñanza, se ha realizado un estudio comparativo de las calificaciones que los alumnos obtuvieron en cinemática durante los últimos cinco años. En los tres primeros cursos (octubre 1988-1990), el desarrollo del tema se lievó a cabo mediante su explicación teórica y la realización de dos prácticas de laboratorio, mientras que en los primeros trimestres de 1991 y 1992 se ha complementado este estudio con la simulación de «movimientos bidimensionales de proyectiles en medios con diferentes resistencias».
Figura 7

Valoracion de los objetivos docentes propuestos en la simulación

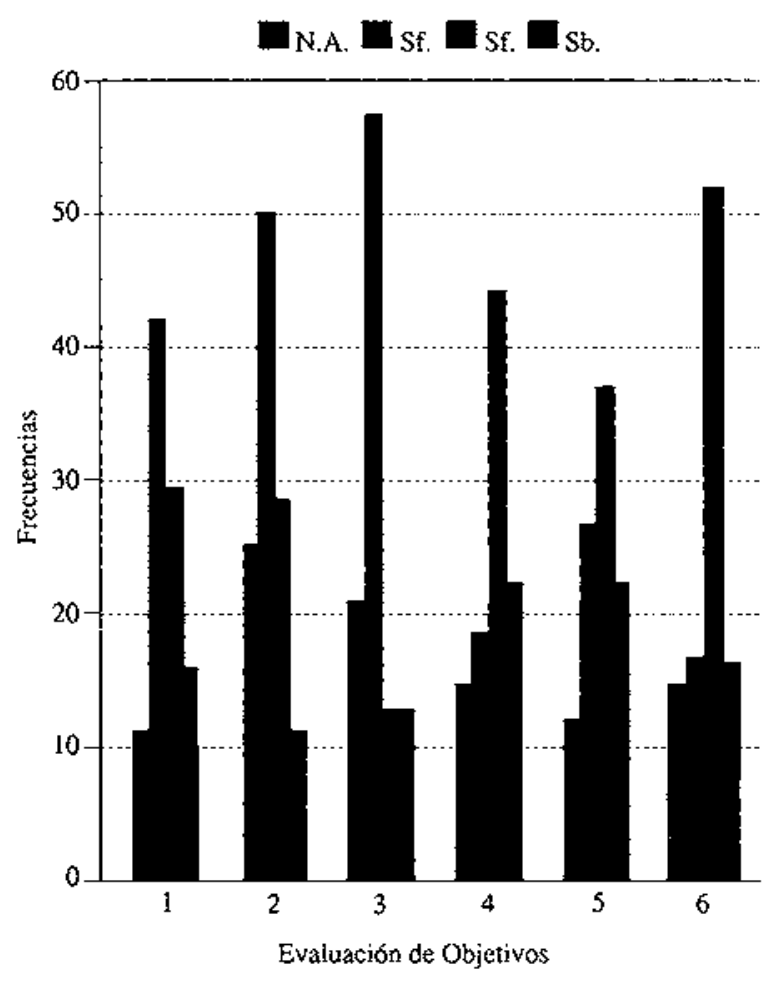

Octubre de 1992

Muestra de 70 alumnos

Los resultados del análisis didáctico comparativo se muestran en la gráfica correspondiente a la figura 8 . En ella, se han representado las frecuencias de incidencia de las diferentes categorías de evaluación de los alumnos (no apto, suficiente, notable y sobresaliente) para los años especificados. Se observa que durante los años 1988 y 1989 las frecuencias de las cuatro categorías se mantienen aproximadamente constantes, permaneciendo éstas en torno al $40 \%$ en suficientes y $50 \%$ en no aptos. En el año 1990 se elevó la calificación de no apto hasta un nivel del $65 \%$, achacable a que durante este período de tiempo se llevó a cabo una huelga intermitente de alumnos que se reflejó en un menor rendimiento académico. En estos dos últimos años, se ha comprobado que el no apto ha bajado hasta un nivel del $25 \%$, el suficiente se ha mantenido en el $40 \%$, pero el notable ha subido en torno al $28-30 \%$.

Para analizar correctamente los resultados obtenidos, ha de tenerse en cuanta otra variable importante, como es la procedencia de nuestro alumnado y que a continuación se va a comentar. En las ingenierías técnicas hasta un $30 \%$ procede de FP, lo que implica la falta de conocimientos básicos necesarios a la hora de abordar la asignatura y que conlleva el no haber conseguido rebajar el $25 \%$ de no aptos. Por otra parte, y al mismo tiempo, existe un $70 \%$ de alumnos que proceden de COU y que además, y debido a la demanda de estas titulaciones, han 
Figura 8

Cráfico comparativo de calificaciones del estudio de cinematica Valoración para los años 1988 al 1992.

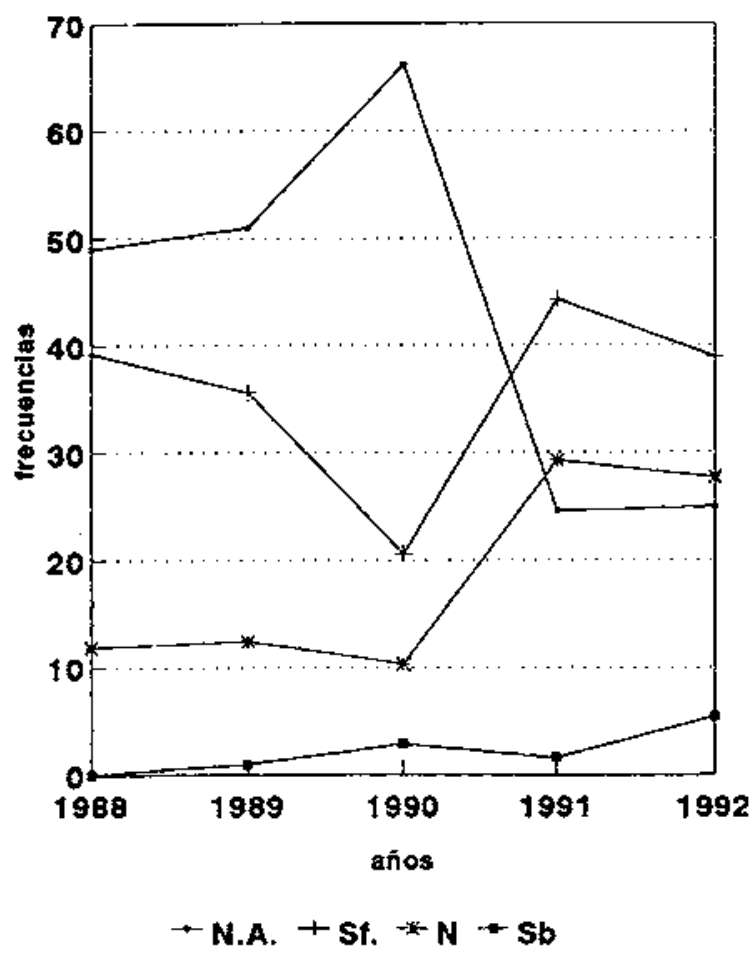

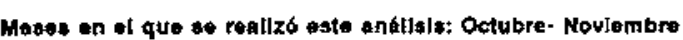

superado la selectividad con una nota media aproximada de 6,0 en los años descritos. Este último porcentaje es sobre el que se analiza el traspaso de las calificaciones de apto a notable. Por último, cabe subrayar que la frecuencia de la calificación máxima permanece aproximadamente constante, debido a los alumnos más destacados que cada año abordan las diversas titulaciones.

\section{REFERENCIAS BIBLIOGRÁFICAS}

BORGHI, L., DE AMBROSI, A. y MASCHERATTI, P., 1987. Computer simulation and laboratory work in the teaching of mechanics, Physis Education, Vol. 22, pp. I17-121.

BORK, A., 1987. El ordenador en la enseñanza. (G. Gili: Barcelona).

CARTIS, F. y GERALD, M., 1987. Applied numerical analysis. (Wesley).

\section{CONCLUSIONES}

En este trabajo se ha tratado de presentar el ordenador como una herramienta didáctica complementaria, pero útil, que ha de integrarse en el proceso de enseñanzaaprendizaje, dadas sus valiosas aplicaciones como sistema de información y, especialmente, por las interesantes posibilidades que ofrece en el campo de la simulación de procesos físicos complejos. Como han indicado otros autores, y hemos tenido oportunidad de comprobar en nuestra experiencia docente, dichos programas de simulación favorecen la participación del alumno en su aprendizaje debido a la interaccion que se produce entre éste y el ordenador. Éste es un aspecto que por sí solo justifica la utilización del mismo como herramienta didáctica que complementa la intervención đel profesor y de otros medios educativos. No hay que olvidar la importante función que desempeña la motivación y la actitud favorable del alumno en el aprendizaje (Gil 1991).

Por otra parte, los resultados analizados anteriormente muestran una tendencia relativamente positiva a favor de los alumnos que han desarrollado la experiencia utilizando el ordenador. Aunque estos resultados no pueden considerarse como definitivos, ya que en el futuro creemos conveniente mejorar el diseño de la investigación y el proceso đe evaluación, nos permiten adoptar una postura moderadamente positiva y continuar perfeccionando nuestra línea de trabajo.

De todo lo expuesto en esta comunicación, se concluye que este tipo de instrumentos didácticos mejora la calidad de las enseñanzas experimentales y, por tanto, el nivel de conocimientos alcanzados por los estudiantes. Por otra parte, pensamos que la utilización didáctica del ordenador se irá generalizando en un futuro próximo, por lo que se hace necesario seguir elaborando materiales didácticos de este tipo e investigando su influencia en Ios procesos de aprendizaje.

DRIVER, R, GUESNE, E. y TIBERGHIEN, A., 1985. Children's ideas in Science. (Milton Keynes, Open University Press). Trad. cast: 1988, Ideas científicas en la infancia y la adolescencia. (Morata: Madrid).

DRIVER, R., 1988. Un enfoque constructivista para el desarrollo del currículo en ciencias, Enseñanza de las Ciencias, Vol. 6(2), pp. $109-120$. 
FRANCO, A., 1991. Física con ordenador. (Universidad del País Vasco: Bilbao).

GIL, D. y CARRASCOSA, J., 1985. Science learning as a conceptual and methodological change, European Journal of Science Education, Vol. 7(3), pp. 231-236.

GIL PEREZ, D., 1986. La metodología científica y la enseñanza de las ciencias: unas relaciones controvertidas, Enseñanza de las Ciencias, VoI. 4(2), pp. 111-121.

GII, D., CARRASCOSA, J., FURIÓ, C. y MARTÍNEZTORREGROSA, I., 1991. La enseñanza de las ciencias en la educación secundaria. (ICE-Horsori: Barcelona).

GORGA, 1., ROSO, C. y VACCA, A.M., 1989. Empleo del computador en la enseñanza del principio fundamental de la dinámica, Enseñanza de las Ciencias, Vol. 7(1), pp. 105-106.

HANCOCK, L. y KRIEGER, M., 1987. Introducción allenguaje C. (McGraw-Hill).

HARTLEY, J.R., 1988. Learning from computer based in learning in science, Studies in Science Education, Vol. 15, pp. 55-76.

HEWSON, P.W, 1990. La enseñanza de fuerza y movimiento como cambio conceptual, Enseñanza de las Ciencias, Vol. $8(2)$, pp. $157-171$

HIERREZUELO, J. y MONTERO, A., 1990. La ciencia de los alumnos. (Elzevir: Vélez-Málaga).

HURLEY, R., 1986. Fisica con LOGO. (Anaya: Madrid).

LOWY, E. y ROSADO, L., 1990. Microordenadores en la enseñanza de las ciencias. (UNED: Madrid).
MARION, J.B., 1975. Dinámica clásica de las partículas y los sistemas. (Reverte: Barcelona).

MARTÍN, E., ZAMARRO, E. y MENÉNDEZ, R., 1988. LOS ordenadores en la enseñanza de la física: la gravitación universal, Revista Española de Física, Vol. 2(1), pp. 46-50.

MARTÍNEZ-JIMÉNEZ, P., POSADILLO SÁNCHEZ DE PUERTA, R., PEDROS-PÉREZ, G. y LEÓN ÁLVAREZ, J., 1992. Introducción mediante simulación de la óptica geométrica, Octava conferencia nacional de física, Vol. 1 , pp. 616-617. (Vila Real: Portugal)

NOVAK, I.D., 1988. Constructivismo humano: un consenso emergente, Enseñanza de las Ciencias, Vol. 6 (3), pp. 213-223.

POSNER, G.J., STRIKE, K.A., HEWSON, P.W. y GERTZOG, W.A., 1982. Accommodation of a scientific conception; Toward a theory of conceptual change, Science Education, Vol. 66 (2), pp. 221-227.

RÍO,L.M.,GONZÁLEZ, J.M., PANIAGUA, J.M., BARRIGÓN, J.M., BAEZA, A. y MIRÓ, C., 1990. Descripción y manejo simulado de un osciloscopio mediante un microordenador, Revista Española de Física, Vol. 4 (1), pp. 72-76.

RÍO, L.M., GONZÁLEZ, J.M., PANIAGUA, J.M., BARRIGÓN, I.M., BAEZA, A. y MIRO, C., 1991. Computer simulated behaviour of a system of molecules in an isolated container, Eur. J. Phys., Vol. 12, pp. 129-134.

VALENTE, M. y NETO, A.J., 1992. El ordenador y su contribución a la superación de dificultades de aprendizaje en mecánica, Enseñanza de las Ciencias, Vol. 10 (1), pp. 80-85.

WILSON, J.H., 1991. Computer software has begun to change physics education, Computer in Physics, Vol. 5 (6), pp. 580-581. 